\title{
Ventilación proporcional asistida: 10 razones para su uso en el destete de ventilación mecánica
}

\author{
Edgar A. Ojeda-Izquierdo ${ }^{1 *}$, John O. Guamán-Crespo ${ }^{1}$, Mijail Frías $^{1}$, Ramón Lozano-Zúñiga ${ }^{2}$ y \\ Arturo Garza-de la Maza ${ }^{3}$ \\ ${ }^{1}$ Unidad de Terapia Intensiva, Hospital San Ángel Inn Universidad; ${ }^{2}$ Unidad de Medicina Crítica Obstétrica, Instituto Nacional de Perinatología \\ Isidro Espinosa de los Reyes; ${ }^{3}$ Unidad de Terapia Intensiva Cardiovascular, Instituto Nacional de Cardiología Ignacio Chávez. Ciudad de México, \\ México
}

\section{Resumen}

Introducción: La búsqueda de modos ventilatorios que disminuyan los efectos adversos, además de mantener los parámetros de protección alveolar, ha sido tema de investigación continua. La ventilación proporcional asistida (VPA) conserva la función muscular diafragmática, coordinando el esfuerzo respiratorio del paciente y el aporte que brinda el ventilador. Presentamos un resumen de la VPA y 10 razones para su uso en el destete de la ventilación mecánica, incluyendo una comparación con el modo de destete más frecuentemente usado: la ventilación con presión soporte (PSV). Métodos: Realizamos una revisión bibliográfica recogiendo información de PubMed, CINAHL y la Biblioteca Cochrane desde 2001 hasta 2019 , para evidenciar los efectos de la VPA en los pacientes ventilados en unidad de cuidados intensivos (UCI). Comparamos VPA vs. PSV como grupo control; observamos que el tiempo de ventilación mecánica invasiva antes de iniciar con el destete fue de 114.98 horas para VPA y 134.56 horas para PSV; destete exitoso en el 88.25 vs. $78.9 \%$, mortalidad en UCl del 6.27 vs. $13.4 \%$ y mortalidad hospitalaria del 19.17 vs. $25.72 \%$ para los grupos VPA y PSV respectivamente. Discusión y conclusiones: Utilizar VPA es segura para los pacientes, mejora de manera significativa el éxito en la extubación, disminuye el tiempo dependiente de ventilador y la mortalidad.

Palabras clave: Ventilación proporcional asistida. Destete de ventilación mecánica. Éxito en la extubación. Mortalidad en UCl. Estancia hospitalaria.

\section{Proportional assist ventilation: 10 reasons for use in weaning from mechanical ventilation}

\begin{abstract}
Introduction: The search for ventilatory modes that reduced adverse effects, in addition to maintaining alveolar protection parameters, has been the subject of continuous research. Proportional assist ventilation (PAV) preserves diaphragmatic muscle function, coordinating the patient's respiratory effort and the input provided by the ventilator. We present a summary of PAV and 10 reasons for its use in weaning from mechanical ventilation, including a comparison with the most frequently used mode of weaning: Pressure Support Ventilation (PSV). Methods: We conduct a literature review collecting information from PubMed, CINAHL and the Cochrane Library from 2001 to 2019, to show the effects of VPA in patients ventilated in the
\end{abstract}

\section{Correspondencia:}

*Edgar A. Ojeda-Izquierdo

E-mail: dr.edgar.ojeda@gmail.com
Disponible en internet: 30-07-2021 Rev Educ Investig Emer. 2021;3(2):78-90 www.medicinadeemergencias.com bajo la licencia CC BY-NC-ND (http://creativecommons.org/licenses/by-nc-nd/4.0/). 
ICU. Results: We compare PAV vs. PSV as a control group; We observed that the time of invasive mechanical ventilation before starting with weaning was 114.98 hours for PAV and 134.56 hours for PSV; Weaning successfully at $88.25 \%$ vs. $78.9 \%$, ICU mortality of $6.27 \%$ vs. $13.4 \%$ and hospital mortality of $19.17 \%$ vs. 25.72 for the PAV and PSV groups respectively. Discussion and conclusions: Using proportional assisted ventilation is safe for patients, significantly improves extubation success, decreases ventilator-dependent time and mortality.

Key words: Proportional assist ventilation. Weaning from mechanical ventilation. Success in extubation. ICU mortality. Hospital stay.

\section{Introducción}

El manejo ventilatorio en escenarios críticos es un tema de importancia creciente, en especial durante cuadros de insuficiencia respiratoria aguda. Un gran problema de la ventilación mecánica invasiva (VMI) son los efectos adversos, entre los que se incluyen sobresedación, atrofia muscular respiratoria y otros derivados de estrategias que no favorecen la protección alveolar, afectando a los pacientes críticos con ventilación mecánica prolongada, lo que supone mayor morbimortalidad, necesidad de vigilancia por personal médico y, por ende, mayor costo hospitalario.

Un efecto lesivo de la VMI es la atrofia del diafragma, que se puede evitar al conservar el esfuerzo respiratorio del paciente durante el mayor tiempo posible. Esto demanda la constante evaluación muscular respiratoria. Los parámetros usados como referencia para dicha evaluación son: el trabajo respiratorio (WOB, work of breathing) y el producto de presión.tiempo, sin embargo, por su dificultad para llevarlos a la práctica clínica son escasamente usados.

Durante la VMI en modo espontáneo, el tórax se encuentra bajo la influencia de dos bombas ventilatorias: una gobernada por el control central del paciente y otra por el ventilador, configurado por el cuidador de acuerdo con su criterio clínico. Los dos sistemas deberían funcionar en armonía para lograr la progresión del destete de la ventilación mecánica. No obstante, cuando el paciente inicia la inspiración, el ventilador insufla gas hasta completar el ciclo inspiratorio, pero la segunda parte de la inspiración puede sobreponerse con la etapa espiratoria neural dada por el paciente, lo que provoca disincronías, ocasionando una pobre interacción entre el paciente y el ventilador, con efectos perjudiciales para el manejo ventilatorio en la insuficiencia respiratoria aguda.

En este trabajo presentamos un resumen de una modalidad ventilatoria, la ventilación proporcional asistida (VPA, proportional assist ventilation) y 10 razones que impactan directa e indirectamente en la retirada de la VMI, así como su comparación con el modo más usado de destete: la ventilación con presión soporte (PSV, pressure support ventilation), para evidenciar las diferencias que existen expresadas en mortalidad, días dependientes de ventilador y éxito de la retirada de la VMI.

\section{Metodología}

Realizamos una revisión bibliográfica con recolección de información de PubMed, CINAHL y la Biblioteca Cochrane desde 2001 hasta 2019, con los siguientes términos de búsqueda: "ventilación proporcional asistida" o "retiro de la ventilación mecánica invasiva" o "ventilación con soporte de presión" o "lesión pulmonar inducida por ventilador" o "destete de la ventilación mecánica" o "éxito en el destete de la ventilación mecánica" o "fracaso en el destete de la ventilación mecánica" o "mortalidad en UCl" o "duración de la estadía" o "días libres de ventilador". No se establecieron restricciones de idioma. Se incluyeron los estudios que brindaron la información de pacientes adultos con ventilación mecánica, de grupos paralelos y cruzados, que examinaron la eficacia de la VPA en comparación con la PSV en adultos con ventilación mecánica. Se excluyeron los estudios que no examinaron los días dependientes de ventilación mecánica, aquellos estudios que no especificaban éxito o fracaso de la retirada de ventilación, los estudios de ventilación pediátrica y no invasiva, así como los grupos paralelos.

La VPA es un modo de VMI que busca coordinar las dos bombas ventilatorias, haciendo que el ventilador quede bajo el control central de la respiración del paciente. Es una forma de asistencia ventilatoria parcial sincronizada, en la que el ventilador genera presión en proporción al esfuerzo espontáneo: mientras más esfuerzo hace el paciente, más apoyo brinda el ventilador mecánico ${ }^{1}$. Es decir, el ventilador amplifica el esfuerzo respiratorio espontáneo del paciente, sin haber seleccionado un respaldo de presión, flujo o volumen por parte del operador y se rige bajo el control completo del impulso ventilatorio del paciente para determinar la profundidad y frecuencia de las respiraciones ${ }^{2}$. 
Fue inventada por Younes, et al. en Norte América en 1992 y desde entonces es objeto de investigación experimental y clínica, gracias a la determinación no invasiva de la resistencia $(R)$ y elastancia $(E)$ del sistema respiratorio, lo que ha permitido ajustar la función del ventilador a la mecánica respiratoria ${ }^{3}$.

\section{¿Cómo se puede entregar presión en proporción al esfuerzo del paciente sin medir directamente dicho esfuerzo?}

La VPA sigue la ecuación de movimiento, uno de los fundamentos de la mecánica respiratoria, que establece que la presión aplicada por los músculos respiratorios al sistema se utiliza para vencer las fuerzas opuestas E y R (Fig. 1A) ${ }^{4}$.

Los alveolos y la pared torácica son representados como un compartimento elástico que se opone a la expansión. La presión de retroceso elástico (PEI) es una función que determina qué tanto volumen pulmonar se desvía desde la capacidad residual funcional (CRF) pasiva y la rigidez del sistema:

\section{$P E I=V \times E$}

Donde $V$ es el volumen por encima de la CRF y $E$ es la elastancia del sistema respiratorio. En un sistema pasivo, la PEl aumenta la presión alveolar (Palv) mientras el pulmón es artificialmente insuflado. Durante la ventilación asistida, los músculos inspiratorios se encuentran activos. Dichos músculos disminuyen la Palv por una cantidad correspondiente a su presión de salida (presión muscular, Pmus).

El compartimiento elástico está conectado hacia las vías aéreas externas mediante el tubo endotraqueal. El ventilador controla la presión en la vía aérea externa (Paw, airway pressure). El flujo de aire hacia los pulmones ocurre cuando la Paw excede la Palv. El flujo es una función de la diferencia de dichas presiones y de la resistencia del tubo, por lo tanto:

$$
\text { Flujo }=\frac{(\text { Paw }- \text { Palv })}{R}
$$

Es decir, la presión generada por los músculos inspiratorios y la vía aérea se disipan para superar la elastancia y la resistencia del sistema respiratorio de acuerdo con la ecuación del movimiento:

$$
\text { Pmus + Paw }=(\vee \times R)+(V \times E)+P E l_{\text {ee }}
$$

Donde $V$ es el flujo instantáneo, $V$ es el volumen instantáneo por encima del volumen final espiratorio, $\mathrm{PEl}_{\mathrm{ee}}$ es la presión de retroceso elástico al final de la espiración (Palv si PEl ee es pasiva; PEEP, si es positiva).
Esta ecuación simplemente establece que la fuerza de distensión es la suma de las fuerzas generadas por el paciente (Pmus) y el ventilador (Paw), y que dicha fuerza de distensión se opone a la suma de la presión resistiva y de $\mathrm{PEI}^{4}$.

El sistema de entrega de gas se observa en la figura 1B. Dicho sistema consiste en un pistón presurizado por un motor lineal de respuesta rápida, que permite el flujo de gas, rápido y libre, en respuesta a cambios en la Palv. La fuerza ejercida por el motor es una función del flujo y el volumen que salen del ventilador. Un esfuerzo mayor resulta en una mayor reducción de la Palv, extrayendo más gas del pistón y resultando en más asistencia ${ }^{5}$.

Las ganancias de las señales de flujo y volumen son ajustables por amplificadores separados: asistencia de flujo (FA) y asistencia de volumen (VA). La salida sumada de los dos amplificadores es la entrada al motor. Por lo tanto, la salida de presión del ventilador es una función del flujo instantáneo y el volumen que salió del ventilador desde su activación. Entonces, con VPA, Paw es la función de flujo y volumen de acuerdo con:

$$
\text { Paw }=(\vee \times F A)+(V \times V A)
$$

FA y VA (cada una tiene unidades diferentes de resistencia y elastancia, respectivamente) deben mantener valores similares, para que la relación Paw-Pmus sea constante y exista proporcionalidad. Entonces:

$$
P a w=K(v \times R+V \times E)
$$

Donde $\mathrm{K}$ es el porcentaje de asistencia, siempre menor al $100 \%$. Al reemplazar Paw en la ecuación del movimiento, obtenemos:

$$
\text { Pmus }=(R-F A) \times V+(E-V A) \times V+P E I_{e e}
$$

Esto indica que con VPA los músculos inspiratorios afrontan la situación después de la activación, con una poscarga que se reducirá en una cantidad igual de FA y VA.

Por lo tanto, la VPA amplifica el esfuerzo ventilatorio del paciente sin imponer ningún objetivo de flujo, volumen o presión.

\section{Proporcionalidad}

Durante la fase inspiratoria, el volumen aumenta progresivamente alcanzando su pico máximo al final de la inspiración. En contraste, el flujo alcanza su pico máximo desde el inicio hasta la mitad de la inspiración y cae posteriormente. Así, la contribución relativa de las presiones elásticas y resistivas varían considerablemente durante la fase inspiratoria. Si el mismo 


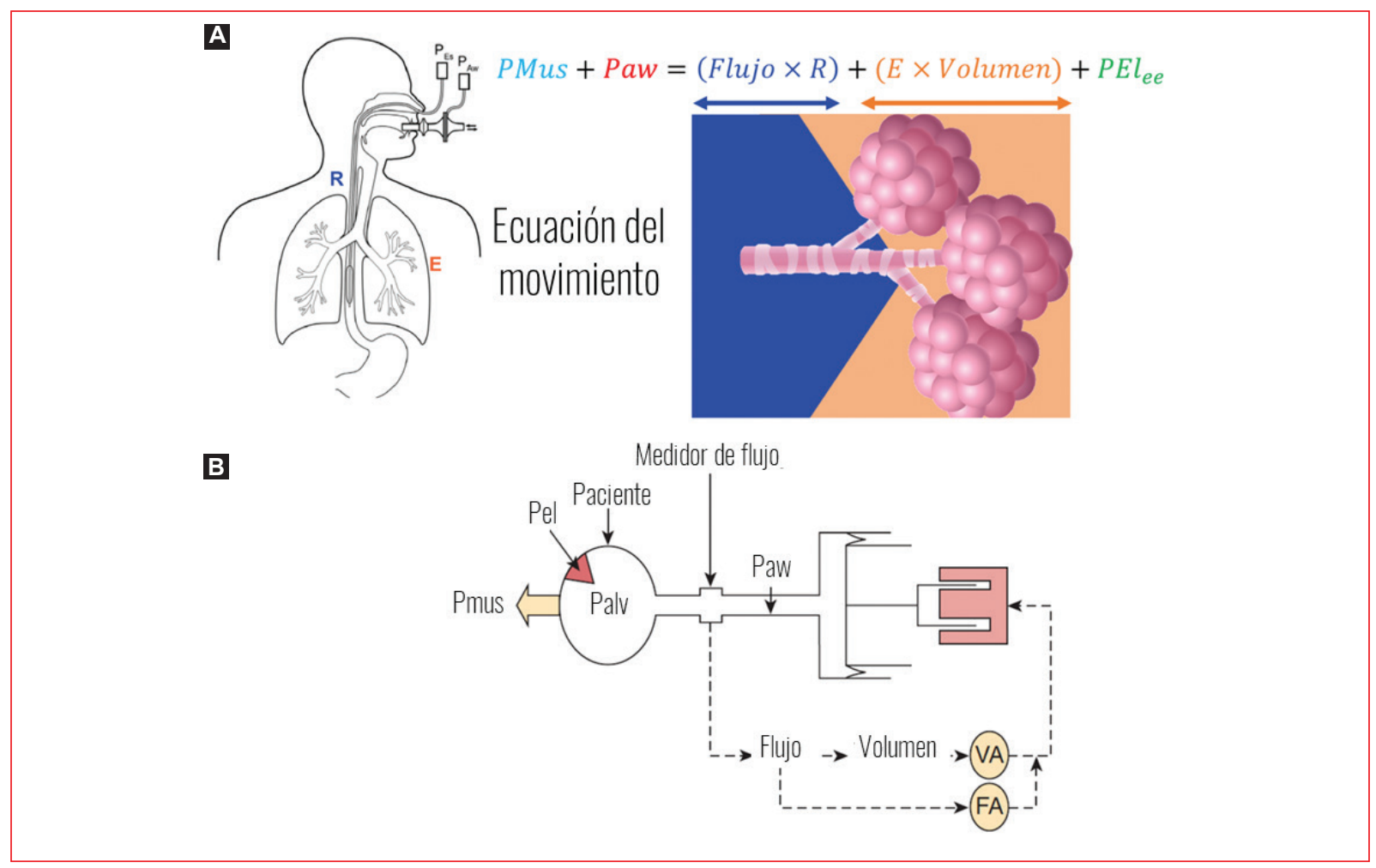

Figura 1. Fisiología de la VPA. A: en los pacientes con ventilación mecánica, la presión total aplicada al sistema respiratorio durante una inspiración es la suma de Pmus y Paw, que se opone a la suma de las presiones resistivas, de retroceso elástico, y de fin de espiración, expresadas en la ecuación del movimiento. B: diagrama de la presión generada por VPA proporcional al flujo y volumen instantáneo, es decir, el sistema genera presión en proporción al esfuerzo del paciente. VPA: ventilación proporcional asistida; Pmus: presión muscular; Pes: presión esofágica; Pel: presión elástica; Paw: presión de la vía aérea; $\mathrm{PEl}_{\mathrm{ee}}$ : presión de retroceso elástico al final de la espiración; Palv: presión alveolar; VA: asistencia de volumen; FA: asistencia de flujo. R: resistencias; E: elastancia.

porcentaje de asistencia (contribución del ventilador) es usado para ambos componentes, la asistencia total (Paw) representa el mismo porcentaje de presión total, a pesar de la contribución relativa de cada uno. Entonces, el porcentaje de asistencia es constante en toda la inspiración, la contribución del paciente (100 - \% asistencia) también es constante en todo momento y la relación entre Paw y Pmus está dada por:

$$
\text { Proporcionalidad }=\frac{\% \text { de asistencia }}{100-\% \text { de asistencia }}
$$

Es decir, si se configuraran tres niveles de asistencia, por ejemplo 20,50 y $80 \%$, cuando se aplica el mismo porcentaje de asistencia a ambos componentes, la forma de la asistencia (Paw) es idéntica a la de la Pmus, pero la proporcionalidad es diferente (Paw/Pmus =0.25, 1.0 y 4.0, respectivamente). Ya que las curvas de Paw y Pmus son idénticas, la disminución de Pmus al final de la espiración está asociada con la disminución en Paw, asegurando sincronía ${ }^{6}$.

\section{Efectos fisiológicos}

Las respuestas del paciente ventilado en VPA pueden estar mediadas por factores químicos (cambios en la tensión de gases sanguíneos) o por factores no químicos del impulso respiratorio.

- Factores químicos: durante el sueño y la anestesia, los factores químicos son la única fuente de impulso respiratorio, por ende, la reducción artificial de la presión parcial de dióxido de carbono $\left(\mathrm{PCO}_{2}\right)$ en estas condiciones elimina los esfuerzos respiratorios 7,8 .

- Factores no químicos: en individuos alerta existen otros factores, que presumiblemente surgen de centros conductuales y de mecanismos respiratorios que operan solo durante la consciencia. 
Los pacientes que requieren ventilación mecánica cubren un amplio espectro de niveles de consciencia. Por lo tanto, es difícil establecer conclusiones generales sobre la aportación de sus impulsos, sin embargo, comentaremos los puntos más importantes en el apartado del impacto con la retirada de la VMI.

\section{¿Cuál es la diferencia fundamental con otros modos espontáneos?}

Con la VPA, la asistencia varía directamente con la intensidad del esfuerzo del paciente. Por el contrario, con PSV la asistencia es la misma, respiración tras respiración, independientemente de la intensidad del esfuerzo (Fig. 2).

En situaciones en las que existe poco esfuerzo ventilatorio, como se observa en la parte baja de la curva de la figura 2A, la PSV ofrece soporte ventilatorio necesario para mantener el WOB dentro de parámetros que permiten la movilidad diafragmática; si el esfuerzo aumenta, el WOB total también, y ya que el soporte no cambia, es el paciente quien asume dicho esfuerzo (obsérvese el tamaño de las letras e iconos que representan la contribución de la PSV y del paciente en la parte alta de la curva), propiciando dificultad respiratoria.

En contraste, la VPA ofrece soporte proporcional en cualquier parte de la curva (Fig. 2B), manteniendo el WOB dentro de límites de protección, y en casos en que incrementa el esfuerzo (ansiedad, taquicardia, doIor), el WOB total aumenta, pero en función del esfuerzo del ventilador y no del paciente (nótese el tamaño de las letras e iconos que representan la contribución de la VPA y del paciente), propiciando el confort y la actividad diafragmática.

Con la VPA, el final del ciclo del ventilador se sincroniza automáticamente con el final del esfuerzo del paciente, mientras que con otros modos no. Con la PSV, la sincronía entre los extremos de las fases inspiratorias del ventilador y del paciente puede o no ocurrir dependiendo de la mecánica respiratoria del paciente y la relación entre el nivel de PSV y Pmus. Los ciclos de ventilación a menudo se extienden más allá del esfuerzo inspiratorio o pueden estar casi completamente desfasados con ellos ${ }^{9}$.

Las características operativas de la asistencia ventiladora ajustada neuralmente (NAVA) son muy similares a las de la VPA en que la asistencia es proporcional al esfuerzo instantáneo en ambos casos. Por lo tanto, la asistencia aumentará cuando aumente el esfuerzo y viceversa, y el ciclo del ventilador terminará poco después del final del esfuerzo inspiratorio en los dos modos. La principal diferencia está en la señal utilizada para conducir el ventilador: la VPA utiliza una estimación indirecta no invasiva del esfuerzo (Pmus calculado), mientras que NAVA utiliza una estimación directa de la actividad diafragmática obtenida de los electrodos esofágicos insertados internamente. Esto le brinda a NAVA una ventaja relativa en cuanto al inicio del esfuerzo inspiratorio, sin embargo, debido a que el diafragma participa en muchas actividades no respiratorias (como cambios posturales y vómitos), el control de la presión de las vías respiratorias mediante la actividad diafragmática podría dar lugar a grandes aumentos indeseables de la presión de las vías respiratorias durante tales actividades ${ }^{10}$.

\section{Impacto con la retirada de la ventilación mecánica}

Mediante la revisión realizada logramos presentar el impacto de VPA en la retirada de la ventilación mecánica, que guarda estrecha relación con los siguientes puntos.

\section{Reducción de los días de estancia en la unidad de cuidados intensivos}

La VMI prolongada se presenta hasta en el $31 \%$ de los pacientes ventilados, incrementando mortalidad, morbilidad, estancia en UCl y costos. Bosma, et al. en 2016 demostraron que los protocolos de VPA en prueba de ventilación espontánea, manteniendo los niveles de soporte más bajo posible sin fatiga respiratoria, son una forma segura y eficaz en la población de alto riesgo o ventilación prolongada sin inducir distrés respiratorio $^{11}$. Este modo ventilatorio mejora la calidad de sueño, influenciando directamente en el tiempo de estancia en $\mathrm{UCl}$ y disminuyendo especialmente el delirium $^{12}$.

En la enfermedad pulmonar obstructiva crónica (EPOC), la VPA mejora las respiraciones espontáneas en un paciente con impulso neural intacto². Elganady, et al. compararon el uso de PSV y VPA en el enfermo crítico con EPOC, encontrando que la tasa de éxito en la retirada de la ventilación mecánica fue favorable al grupo de VPA, (90 vs. $66.7 \% ; p=0.028$ ). Los días libres de ventilación mecánica y estancia en UCI fueron significativamente menores en el grupo de VPA?

\section{Reducción de asincronías}

El grupo de trabajo de Elganady, et al. encontró menor índice de asincronías en el grupo de VPA en 


\section{A}

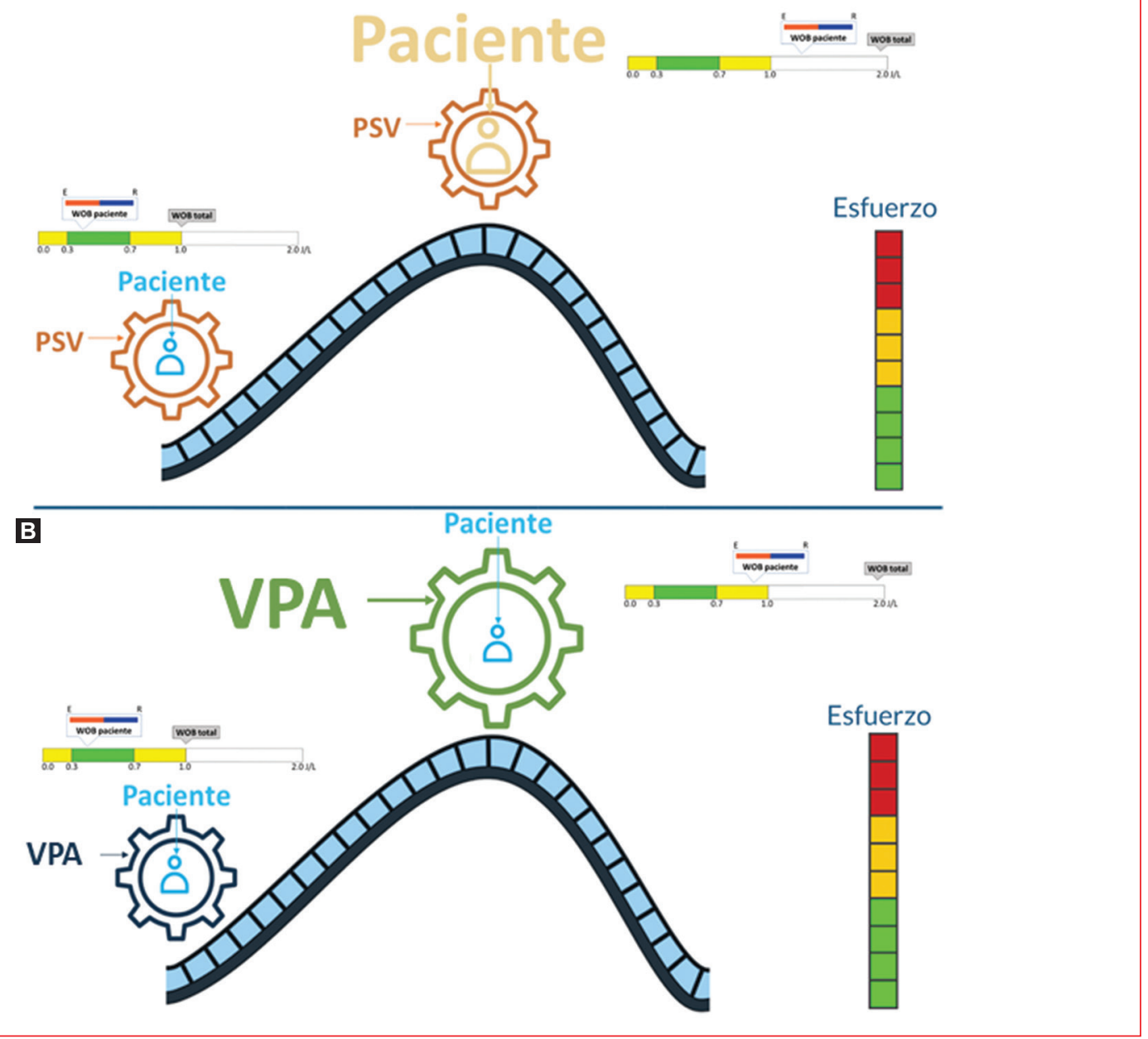

Figura 2. Diferencias entre PSV y VPA. Representación del esfuerzo del paciente y WOB total y del paciente. A: PSV. Nótese que en la parte baja de la curva (poco esfuerzo) existe un WOB total y WOB del paciente que se mantienen dentro de parámetros que permiten el movimiento diafragmático. Al aumentar el esfuerzo (taquicardia, dolor, ansiedad), el WOB total aumenta a costa del WOB del paciente, representado por el tamaño del icono y letras, ya que la presión soporte se mantiene fija. B: VPA. De igual manera, cuando hay poco esfuerzo, el diafragma permanece activo. Cuando se encuentra en la parta alta de la curva, el WOB total aumenta, con poca modificación en el WOB del paciente. A diferencia de la PSV, el ventilador se encarga de suplir este trabajo, representado por el tamaño del icono y letras. WOB: (work of breathing) trabajo respiratorio; PSV: ventilación con presión soporte; VPA: ventilación proporcional asistida.

relación al de PSV, además de que, en el último grupo, la asincronía de disparo inefectivo se presentó en mayor proporción ${ }^{2}$.

Las asincronías ocurren en la PSV por incapacidad de los pacientes para compensar un alto nivel de presión soporte. Volumen tidal (Vt) alto puede aplicarse la cantidad de Vt no necesariamente depende de la cantidad de esfuerzo realizado en la inspiración, sino en de la cantidad de presión soporte aplicada, y de compliance y resistencia. Esta entrega de volumen tidal alto puede predisponer a asincronías que dependen del paciente (hiperinsuflación dinámica) o del ventilador (esfuerzo inefectivo por exceso de soporte).

En modo de VPA incluso cuando existe una asistencia alta se logra mantener una presión meseta por debajo de los rangos de seguridad, esto probablemente debido a los reflejos neurales (p. ej., Hering-Breuer) que inhiben la actividad muscular inspiratoria si la distensión pulmonar excede el umbral de la capacidad pulmonar total.

La VPA permite adaptarse continuamente al nivel de asistencia requerido de acuerdo con los cambios en la 
mecánica ventilatoria, permitiendo mejoría en la sincronía paciente-ventilador, adaptándose a la impedancia del sistema respiratorio ${ }^{11}$.

\section{Compensa los cambios en la mecánica respiratoria}

La VPA por sus principios fisiológicos de la mecánica respiratoria mide la capacidad de carga y descarga de los músculos respiratorios en una relación estrecha y proporcional al esfuerzo del paciente, promoviendo la actividad diafragmática a lo largo de cada ciclo respiratorio ${ }^{13}$.

El éxito de la compensación dinámica radica en la capacidad de un modo en modificar la demanda otorgada al paciente con base en los requerimientos de volumen corriente ${ }^{14,15}$, por lo cual la superioridad de la VPA radica en la teoría efectiva y corroborada donde la VPA monitorea constantemente la demanda inspiratoria del paciente durante el esfuerzo inspiratorio por medio del flujo y por lo tanto administrando Vt variable. Flujo y volumen es la mezcla administrada que se combina para proporcionar la presión mínima necesaria en cada esfuerzo inspiratorio ${ }^{16,17}$.

\section{Mejora el compromiso de los músculos respiratorios}

Si se conoce el nivel de asistencia, el esfuerzo muscular se puede calcular a partir de la diferencia entre presión máxima de la vía aérea y PEEP. A partir de la ecuación del movimiento la modalidad de VPA calcula la señal que representa una estimación de la Pmus inspiratoria total utilizando valores estimados de resistencia y elastancia del sistema respiratorio ${ }^{18}$. Durante la VPA la Pmus máxima se puede estimar a partir de la presión máxima de la vía aérea, así como su porcentaje de asistencia, generando esfuerzo muscular respiratorio del paciente para favorecer una rehabilitación oportuna donde el paciente genere respiraciones espontáneas que le permitan limitar el desarrollo de atrofia muscular diafragmática y un destete más fácil ${ }^{11,19}$. Esto se da principalmente cuando el ajuste de ganancia de soporte se encuentra en el rango de 5 a $10 \mathrm{cmH}_{2} \mathrm{O}$ de presión muscular pico (Pmus, Pico), permitiendo una adecuada dinámica y mecánica respiratoria ${ }^{20}$.

\section{Limitación de la presión de distensión}

El Vt es la variable que se debe controlar durante la VMI, con la finalidad de evitar lesión inducida por el ventilador. Sin embargo, estudios recientes en pacientes con síndrome de dificultad respiratoria aguda (SDRA) mostraron que las reducciones en $\mathrm{Vt} O$ aumentos en PEEP pueden incrementar la supervivencia solo si existe una disminución en la presión de distensión (presión meseta - PEEP, $\triangle \mathrm{P}$ ). $\Delta \mathrm{P}$ refleja el grado de estiramiento pulmonar durante la ventilación controlada por volumen. Incrementos por encima de $15 \mathrm{cmH}_{2} \mathrm{O}$ en $\Delta \mathrm{P}$ se asocian con disminución en la supervivencia ${ }^{14,21}$.

Estudios de VPA y $\Delta \mathrm{P}^{13}$ demuestran un incremento de la distensibilidad, lo que permite incrementos del Vt sin comprometer la presión de distensión. Pacientes con respiración espontánea incrementan su distensibilidad y por consiguiente se incrementa el Vt. Un mecanismo que puede explicar los cambios en la distensibilidad es la ventilación disminuida en regiones pulmonares que se encuentran cerca del diafragma durante la ventilación controlada. La contracción diafragmática redistribuye la ventilación en estas zonas, y de este modo favorece el reclutamiento, teniendo como resultado final un incremento en la distensibilidad ${ }^{22}$.

\section{Disminución del daño respiratorio}

El daño respiratorio está asociado a la duración de la ventilación mecánica, ya sea por el riesgo de atrofia muscular, la capacidad limitada para una adaptación de Vt y la interacción paciente-ventilador.

Al disminuir el aporte constante de presión positiva en rangos altos en la vía aérea, se disminuye la presión de distensión de esta, obteniéndose menor variabilidad en la distensión alveolar y menos atelectrauma; la disminución de esta variable se ha demostrado que disminuye la mortalidad, además de los días dependientes de ventilador, las infecciones asociadas y las complicaciones propias de un paciente con movilidad limitada. En general podemos comentar que menor cantidad de barotrauma, atelectrauma y biotrauma es notable en estos pacientes. La VPA permite la adaptación continua, evitando el daño respiratorio asociado a la VMI. El proceso de retirada de la ventilación mecánica en un pulmón mínimamente lesionado va a dar como resultado el aumento de la tasa de éxito al retirar el apoyo respiratorio ${ }^{11}$.

\section{Reducción de la disnea}

Se define como disnea a la presencia de una frecuencia respiratoria $(\mathrm{FR})>35$ respiraciones/minuto durante más de 5 minutos, con alguna otra alteración como incremento de la frecuencia cardiaca $>20 \%$ con 
respecto a la basal, uso marcado de los músculos accesorios y/o presencia evidente de diaforesis en pacientes conscientes.

Con la VPA, una disminución de la FR después de haber incrementado el porcentaje de asistencia indica que el paciente es incapaz de mantener un $\mathrm{Vt}$ adecuado, a expensas de FR altas. Si la FR no se modifica con cambios en los niveles de asistencia ventilatoria, representa a la FR espontánea dada por el sistema respiratorio del paciente. En conclusión, es importante observar la FR al disminuir el porcentaje de asistencia, ya que estos cambios pueden indicarnos dificultad respiratoria ${ }^{7,23}$.

Bosma, et al. demostraron el impacto fisiológico de la VPA en la retirada ventilatoria debido a la reducción en las presiones pico de las vías respiratorias y disminución de la asistencia ventilatoria, al impartir una mayor parte de la carga de trabajo respiratorio al diafragma sin inducir signos clínicos de dificultad respiratoria y disminuyendo número de asincronías paciente-ventilador, inclusive con altos niveles de soporte ventilatorio ${ }^{11}$.

\section{Disminución de la carga de trabajo respiratorio}

Se estudiaron 13 pacientes adultos con intubación endotraqueal por insuficiencia respiratoria aguda ${ }^{5,24}$. Se concluyó que la VPA mejoró el patrón de respiración y redujo el esfuerzo muscular inspiratorio en comparación con la respiración espontánea. De igual manera observaron que la adición de asistencia de flujo redujo significativamente el trabajo total de la respiración (debido a una reducción en el componente resistivo), proporcionando evidencia para el uso de asistencia de flujo y volumen en la ventilación.

Grasso, et al. publicaron en el año $2000^{25}$ un estudio que demuestra que en pacientes bajo ventilación mecánica en proceso de retirada, la estrategia utilizada para compensar un aumento agudo de la impedancia respiratoria difiere notablemente entre PSV y VPA. AI incrementarse la impedancia respiratoria, la PSV requirió un mayor esfuerzo muscular (medido por la presión ejercida por el diafragma por minuto y por litro) y causó un malestar más pronunciado en el paciente en comparación con la VPA ${ }^{26}$.

Ranieri, et al. concluyeron que la VPA ofrece una mejor adaptación al esfuerzo del paciente, mediante variabilidad en el Vt y menor esfuerzo muscular medido por presión diafragmática ${ }^{27}$.

\section{Mejora la eficiencia del sueño}

En los pacientes críticamente enfermos es frecuente observar anormalidades del sueño, predominantemente disminución de la fase REM (movimiento ocular rápido) y despertares más frecuentes. Esta alteración está presente aún en pacientes sedados con Ramsay de 3 puntos ${ }^{28}$.

Alexopoulou, et al. demostraron en pacientes sedados que la eficiencia del sueño fue levemente mejor en VPA en contraposición con PSV29. Además, el número de oclusiones de la vía aérea al final de la inspiración, necesarias para las mediciones de la elastancia y resistencia en VPA, no afectaron la calidad del sueño, ya que son de corta duración y no son un factor de importancia para la interrupción y calidad del sueño en pacientes críticos.

En otro estudio se mostró que las asincronías juegan un papel importante en la calidad de sueño. Al comparar PSV y VPA, observaron menores interrupciones del sueño, que representaron el 24 y el $10 \%$, respectivamente ${ }^{30}$.

\section{Asistencia con el ejercicio}

El ejercicio temprano en pacientes críticamente enfermos es seguro y beneficioso para preservar la fuerza muscular, esto se logra iniciando una rehabilitación temprana, disminuyendo la mortalidad ${ }^{31,32}$.

Akoumianaki, et al. en $2017^{31}$ evidenciaron que los pacientes en modo PSV mostraron un claro incremento del consumo de oxígeno $\left(\mathrm{VO}_{2}\right)$ en comparación con pacientes en modo VPA. El $\mathrm{VO}_{2}$ no se modificó en ningún momento durante la terapia de ejercicio, de igual manera, en VPA no se observaron cambios significativos en la hemodinamia y en las variables del patrón respiratorio. Otro punto a favor del VPA en este estudio es que la disnea no se vio influenciada por el $\mathrm{VO}_{2}$, más bien en relación con el impulso neural respiratorio (en VPA se preserva mejor) y también en función del aumento de la producción de $\mathrm{VCO}_{2}$.

\section{Programación}

El objetivo de rango de esfuerzo respiratorio es el producto de la Pmus-tiempo (PTPmus). Esta no se puede medir a la cabecera del paciente, por lo que se utiliza su principal subrogado: la Pmus, Pico, que es el máximo movimiento realizado por los músculos inspiratorios durante la inspiración. Dicho valor se estima según la ecuación: 
Pmus, Pico $=($ PPico - PEEP $) \times\left(\frac{100-\text { Ganancia }}{\text { Ganancia }}\right)$

El valor para mantener una adecuada función diafragmática se encuentra entre $5-10 \mathrm{cmH}_{2} \mathrm{O}$ de Pmus, $\mathrm{Pico}^{33}$. Asumiendo que la curva de Pmus tiene una forma triangular, con el final del esfuerzo inspiratorio en la Pmus, Pico, la PTPmus, que representa el área bajo la curva de Pmus durante el tiempo inspiratorio (Tinsp), puede ser estimada en un minuto (en $\mathrm{cmH}_{2} \mathrm{O} \cdot \mathrm{s} / \mathrm{min}$ ) según la ecuación:

$$
\text { PTPmus }=\left(\frac{\text { Pmus, } \text { Pico } x \text { Tinsp }}{2}\right) \times F R
$$

En el estudio de Carteaux ${ }^{19}$ se consideraron los valores usuales de Tinsp y de FR, apuntando que valores de Pmus, Pico de 5-10 $\mathrm{cmH}_{2} \mathrm{O}$ se encontrarían dentro de valores de PTPmus de $50-150 \mathrm{cmH}_{2} \mathrm{O} \cdot \mathrm{s} / \mathrm{min}$. Entonces se realizó una cuadrícula en la que se compararon los valores de ganancias y de delta de presión (PPico - PEEP) necesarios para un determinado valor de Pmus, Pico, resaltando las áreas de Pmus, Pico objetivo. Construimos una tabla, asumiendo el valor objetivo de Pmus, Pico antes mencionado, para correlacionar el cambio ( $\Delta$, delta) de presión PPico - PEEP y las ganancias, con el objetivo de abreviar la cuadrícula original y comparar rápida y eficazmente los niveles de PPico - PEEP y ganancias que se encuentran dentro del objetivo Pmus, Pico $\left(5-10 \mathrm{cmH}_{2} \mathrm{O}\right)$ aproximadamente.

Iniciamos con la configuración inicial, como se observa en la figura 3, con las ganancias al $50 \%$, el disparo inspiratorio a $1 \mathrm{l} / \mathrm{min}$, introducimos el peso del paciente, el número de diámetro del tubo endotraqueal y la presión máxima de la vía aérea, ajustando parámetros de alarmas de la siguiente manera: Paw máxima $40 \mathrm{cmH}_{2} \mathrm{O}$, FR máxima 40 ciclos/minuto, Vt máximo $10 \mathrm{ml} / \mathrm{kg} /$ peso predicho, Vt mínimo $0 \mathrm{ml}$, Flujo máximo $20 \mathrm{l} / \mathrm{min}$, Flujo mínimo $7 \mathrm{l} / \mathrm{min}$, nuestro delta PPico - PEEP debería oscilar entre 5 y $10 \mathrm{cmH}_{2} \mathrm{O}$ (no confundir con valor objetivo de Pmus, Pico).

Debemos calcular la Pmus, Pico y alcanzar el objetivo. Si se encuentra en niveles inferiores, debemos disminuir las ganancias en pasos de un 10\% hasta alcanzarlo. Así mismo, si la Pmus, Pico se encuentra en niveles superiores, se debe aumentar las ganancias en pasos de un $10 \%$. Si nos mantenemos en valores meta, no modificaremos las ganancias, a no ser que existan dos circunstancias: alcalosis respiratoria/Vt $>10 \mathrm{ml} / \mathrm{kg} /$ peso predicho sin causa obvia de hiperventilación, o signos clínicos de dificultad respiratoria/Vt
$<5 \mathrm{ml} / \mathrm{kg} /$ peso predicho/acidosis respiratoria, con lo que debemos disminuir y aumentar las ganancias en pasos de un $10 \%$, respectivamente.

Ajustamos $\mathrm{PEEP}$ y $\mathrm{FiO}_{2}$ de acuerdo con las necesidades de cada paciente; aún no se ha establecido la modalidad ideal para establecer el PEEP ideal, la meta es obtener una saturación capilar de $\mathrm{O}_{2}$ mayor del $88 \%$ o $\mathrm{PaO}_{2}$ mayor de $60 \mathrm{mmHg}$.

\section{Consideraciones}

Valorar el Vt que resulta de la configuración del ventilador, evitando niveles mayores de $8 \mathrm{ml} / \mathrm{kg} /$ peso predicho. Aunque con gran frecuencia el Vt es cercano a $3 \circ 4 \mathrm{ml} / \mathrm{kg} /$ peso predicho, eso no es insuficiente para el paciente, siempre y cuando no haya datos de incomodidad o retención de $\mathrm{CO}_{2}{ }^{33}$.

Al cambiar al paciente a VPA puede aumentar la concentración arterial de $\mathrm{CO}_{2}$, esto se debe probablemente a que en el modo anterior al VPA, el paciente tenía demasiado apoyo; solamente nos debemos preocupar si hay hipercapnia permisiva. Esta parte se refiere al Vt, que dicho en el ejemplo, puede ser excesivo en un modo previo al vpa; al cambiarlo, puede ser más óptimo y ocurrir menor cantidad de Vt puede movilizar el $\mathrm{CO}_{2}$ en menor cantidad, y ocasionar hipercapnia, sin embargo, introducimos el concepto de hipercapnia permisiva al mencionar que la preocupación es si el ph es menor de 7.25.

Los flujos máximos muy altos pueden aumentar la carga resistiva con compensación incompleta, ya que las mediciones de resistencia en la actualidad no son óptimas ${ }^{30}$.

Se podría realizar una prueba de ventilación espontánea con un porcentaje de ganancia menor del 20\% y sin otros signos clínicos de insuficiencia respiratoria.

Se debe considerar cambiar a otro modo ventilatorio si los valores de ganancias son superiores al $85 \%$, si la fracción inspiratoria de oxígeno es $>70 \%$ y si el PEEP es $>10 \mathrm{cmH}_{2} \mathrm{O}$.

\section{Indicaciones y limitaciones}

La VPA está potencialmente indicada en todas las situaciones, exceptuando lo siguiente:

- Depresión respiratoria: para el uso seguro de VPA, el gasto muscular respiratorio del paciente debe responder a los cambios en $\mathrm{PaCO}_{2}, \mathrm{PaO}_{2}$ y $\mathrm{pH}$. Por esto, contraindicación absoluta incluye apnea central, esfuerzos muy débiles y sin dificultad respiratoria a pesar de un $\mathrm{pH}$ anormalmente bajo. 


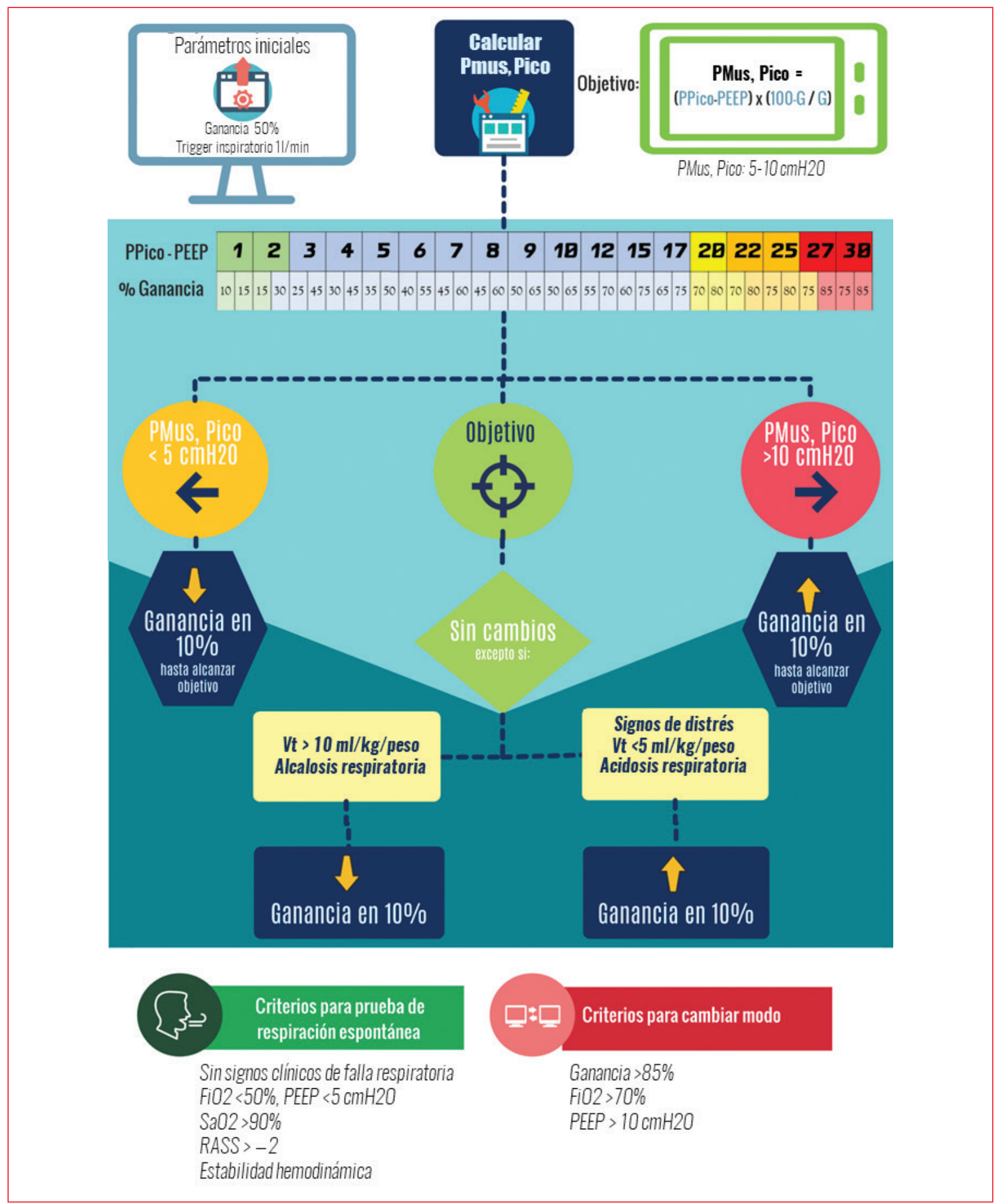

Figura 3. Algoritmo de programación y manejo de VPA. Se configuran parámetros iniciales del modo ventilatorio. Se calcula Pmus, Pico para alcanzar objetivo entre 5-10 $\mathrm{cmH}_{2} \mathrm{O}$. Deben de disminuirse las ganancias si existen valores inferiores y aumentarse si hay valores superiores, en pasos del 10\% en 10\% hasta alcanzar el objetivo, manteniendo dichas ganancias sin modificar y justificando su modificación si existe alcalosis o acidosis respiratoria y otros signos de distrés. Nótese la tabla inicial de delta de presiones (PPico - PEEP) y ganancias, diseñado para mostrar los niveles relacionados entre sí y con niveles de Pmus, Pico óptimos; además, el código de color nos ayuda a reconocer quiénes se podrían beneficiar de una prueba de ventilación espontánea (en verde) y quiénes deberían cambiarse a otro modo (en rojo). Pmus, Pico: presión muscular pico; PPico: presión pico; PEEP: presión positiva al final de la espiración; G: \% de ganancias; Vt: volumen tidal; $\mathrm{FiO}_{2}$ : fracción de oxígeno inspirado; $\mathrm{SaO}_{2}$ : saturación arterial de oxígeno; RASS: escala de sedación y agitación de Richmond. 
Tabla 1. Evidencia VPA vs. PSV. Análisis de VPA vs. PSV en pacientes sometidos a ventilación mecánica invasiva, su retirada y mortalidad

\begin{tabular}{|c|c|c|c|c|c|c|}
\hline \multicolumn{7}{|c|}{ Tiempo de ventilación antes de PVE } \\
\hline Autores & \multicolumn{3}{|c|}{ VPA (horas) } & \multicolumn{3}{|c|}{ PSV (horas) } \\
\hline Botha, et al. ${ }^{34}$ & \multicolumn{3}{|c|}{84.3} & \multicolumn{3}{|c|}{135.9} \\
\hline Bosma, et al. ${ }^{11}$ & \multicolumn{3}{|c|}{93.6} & \multicolumn{3}{|c|}{117.6} \\
\hline Teixeira, et al. & \multicolumn{3}{|c|}{133} & \multicolumn{3}{|c|}{143.3} \\
\hline Sasikumar, et al. & \multicolumn{3}{|c|}{168} & \multicolumn{3}{|c|}{180} \\
\hline Xirouchak, et al. & \multicolumn{3}{|c|}{96} & \multicolumn{3}{|c|}{96} \\
\hline Promedio & \multicolumn{3}{|c|}{114.98} & \multicolumn{3}{|c|}{134.56} \\
\hline \multicolumn{7}{|c|}{ Destete exitoso } \\
\hline \multirow[t]{2}{*}{ Autores } & \multicolumn{3}{|c|}{ VPA } & \multicolumn{3}{|c|}{ PSV } \\
\hline & Total (n) & Éxito (n) & $\%$ & Total (n) & Éxito (n) & $\%$ \\
\hline Botha, et al. & 25 & 23 & 92 & 24 & 19 & 79.16 \\
\hline Bosma, et al. & 27 & 24 & 88.88 & 23 & 18 & 78.26 \\
\hline Teixeira, et al. & 48 & 38 & 79.16 & 46 & 32 & 69.56 \\
\hline Sasikumar, et al. & 13 & 12 & 92.30 & 10 & 9 & 90 \\
\hline Xirouchak, et al. & 108 & 96 & 88.88 & 100 & 78 & 78 \\
\hline Promedio & & & 88.25 & & & 78.99 \\
\hline \multicolumn{7}{|c|}{ Mortalidad en UCI } \\
\hline \multirow[t]{2}{*}{ Autores } & \multicolumn{3}{|c|}{ VPA } & \multicolumn{3}{|c|}{ PSV } \\
\hline & Total (n) & Fallecidos (n) & $\%$ & Total (n) & Fallecidos (n) & $\%$ \\
\hline Botha, et al. & 25 & 1 & 4 & 24 & 6 & 25 \\
\hline Bosma, et al. & 27 & 4 & 14.81 & 23 & 3 & 13.04 \\
\hline Teixeira, et al. & 48 & 0 & 0 & 46 & 1 & 2.17 \\
\hline Promedio & & & 6.27 & & & 13.40 \\
\hline \multicolumn{7}{|c|}{ Mortalidad hospitalaria } \\
\hline \multirow[t]{2}{*}{ Autores } & \multicolumn{3}{|c|}{ VPA } & \multicolumn{3}{|c|}{ PSV } \\
\hline & Total (n) & Fallecidos (n) & $\%$ & Total (n) & Fallecidos (n) & $\%$ \\
\hline Botha, et al. & 25 & 9 & 36 & 24 & 6 & 25 \\
\hline Bosma, et al. & 27 & 3 & 11.11 & 23 & 9 & 39.13 \\
\hline Teixeira, et al. & 48 & 5 & 10.41 & 46 & 6 & 13.04 \\
\hline Promedio & & & 19.17 & & & 25.72 \\
\hline
\end{tabular}

VPA: ventilación proporcional asistida; PVE: prueba de ventilación espontánea; PSV: ventilación con presión soporte; UCI: unidad de cuidados intensivos.

- Alta necesidad de sedación/convulsiones: por la respuesta respiratoria impredecible.

- Debilidad neuromuscular grave: ya que estos pacientes presentan dificultad para iniciar el esfuerzo inspiratorio, y cuando ocurre puede estar cerca del final de este. Como el ciclo del ventilador termina al final del esfuerzo inspiratorio, el ciclo de inflado puede ser muy breve y la ventilación puede ser inadecuada.

- Fístulas broncocutáneas: por la probabilidad de fuga del sistema, si el ventilador no está equipado con algoritmos de compensación automatizada. 
En términos generales, la VPA no funciona correctamente en pacientes con hiperinflación dinámica severa, debilidad muscular respiratoria grave ni en pacientes inconscientes.

\section{Discusión}

En diferentes publicaciones se ha demostrado superioridad de la VPA respecto a la PSV en cuanto a mejoría entre la sincronización, adaptabilidad y calidad del sueño. Sin embargo, existió limitación de estos estudios, ya que la cantidad reducida de pacientes incluidos, protocolos de corta duración en el proceso de destete, así como uso de software de primera generación de la VPA que requerían monitorización manual de la distensibilidad y resistencias durante la ventilación mecánica propició a que no fuera una herramienta útil de cabecera.

Hoy en día se cuenta con el sistema automatizado de circuito cerrado llamado "VPA con factor de ganancia ajustable», que de forma automática, cíclica y no invasiva censa constantemente distensibilidad y resistencia, siendo así más accesible la monitorización para uso clínico de forma sistemática ${ }^{11}$. Estudios aleatorizados demostraron que la tasa de fracaso en el destete, definida como la necesidad de modalidades controladas, fue significativamente menor en VPA que en PSV (11.1 vs. $22.0 \%$; $p=0.041$ ), dentro de las primeras 48 horas $^{12}$.

El final del ciclo ventilatorio de VPA siempre se produce durante la fase de disminución de la presión transdiafragmática, al igual que durante la respiración espontánea, y no se extendió más allá el punto donde dicha presión alcanzó la línea de base, sin comprometer el tiempo espiratorio. Se ha visto que esto disminuye el soporte ventilatorio, aumenta la comodidad del paciente y disminuyen las disincronías.

Luego de analizar cinco estudios que compararon el comportamiento de los pacientes ventilados en VPA y PSV (Tabla 1), se puede evidenciar que antes de someterse al proceso de retirada de la ventilación espontánea, los pacientes manejados con VPA estuvieron 114.98 horas bajo VMI, mientras que los que estaban en modo PSV lo estuvieron por 134.56 horas. Este tiempo expresa que, a pesar de que los pacientes estaban sometidos a ventilación mecánica controlada (al menos de forma inicial y con ventilación espontánea esporádica), existió una disminución del tiempo de dependencia del ventilador en los pacientes del grupo de VPA, tal vez dado por mayor actividad del músculo diafragmático y por los demás factores expuestos anteriormente.
Al someter a los pacientes al protocolo de extubación, se observa que el mayor éxito se obtuvo en el grupo de VPA. Así mismo, en los pacientes que estaban sometidos a la VPA, se observa menor mortalidad en $\mathrm{UCl}$ con respecto a PSV, 6.27 vs. $13.4 \%$ respectivamente, y en cuanto a mortalidad hospitalaria, se evidencia mortalidad del $19.1 \%$ con VPA vs. $25.7 \%$ con PSV. Hay que tener en cuenta que en los ítems de mortalidad se deben de considerar los factores distractores, como la distribución de los diagnósticos en los grupos, la gravedad de los pacientes y los parámetros ventilatorios fijados para cada paciente, ya que en cada terapia intensiva fueron diferentes. De todas maneras, se aprecia la tendencia hacia la disminución de la mortalidad.

\section{Conclusiones}

La VPA es un modo único que mide la capacidad de carga y descarga de los músculos respiratorios en una relación proporcional al esfuerzo del paciente, que podemos utilizar en pacientes con ventilaciones espontáneas, especialmente aquellos con lesiones pulmonares mínimas que condicionen poca presión de distensión, ya que es segura, mejora probabilidad de éxito en la extubación, tiempo dependiente de ventilador y mortalidad.

Este modo ventilatorio está ganando lentamente aceptación entre los médicos, sin embargo, el obstáculo principal sigue siendo la falta de familiaridad con su manejo, así como dogmas sobre cómo debe ser la respiración durante la ventilación mecánica. Esperamos que esta descripción ayude a este respecto.

\section{Financiamiento}

La presente investigación no ha recibido ninguna beca específica de agencias de los sectores público, comercial o sin ánimo de lucro.

\section{Conflicto de intereses}

Los autores declaran no tener conflicto de intereses.

\section{Responsabilidades éticas}

Protección de personas y animales. Los autores declaran que para esta investigación no se han realizado experimentos en seres humanos ni en animales.

Confidencialidad de los datos: Los autores declaran que en este artículo no aparecen datos de pacientes. 


\section{Derecho a la privacidad y consentimiento infor- mado: Los autores declaran que en este artículo no aparecen datos de pacientes.}

\section{Bibliografía}

1. Hosking B, Nikoo M, Bosma KJ. The incidence of patient-ventilator asynchrony during weaning from mechanical ventilation: $A$ comparison of proportional assist ventilation (PAV) to pressure support (PS). Am J Respir Crit Care Med. 2011;183:A3197.

2. Elganady AA, Beshey BN, Abdelaziz AAH. Proportional assist ventilation versus pressure support ventilation in the weaning of patients with acute exacerbation of chronic obstructive pulmonary disease. Egypt J Chest Dis Tuberc. 2014;63(3):643-50.

3. Younes M, Webster K, Kun J, Roberts D, Masiowski B. A method for measuring passive elastance during proportional assist ventilation. Am J Respir Crit Care Med. 2001;164(1):50-60.

4. Younes M, Puddy A, Roberts D, Light RB, Quesada A, Taylor K, et al. Proportional assist ventilation: Results of an initial clinical trial. Am Rev Respir Dis. 1992:145:121-9.

5. Akoumianaki E, Kondili E, Georgopoulos D. Proportional assist ventilation. Eur Respir Monogr. 2012;55:97-115.

6. Younes M, Georgopoulos D. Control of breathing relevant to mechanical ventilation. En: Marini J, Slutsky A, editores. Physiological basis of ventilatory support, lung biology in health and disease. Nueva York, NY: Marcel Dekker; 1998. pp.1-74.

7. Meza S, Mendez M, Ostrowski M, Younes M. Susceptibility to periodic breathing with assisted ventilation during sleep in normal subjects. J Appl Physiol. 1998;85(5):1929-40.

8. Fink BR. Influence of cerebral activity in wakefulness on regulation of breathing. J Appl Physiol. 1961;16:15-20.

9. Yamada Y, Du HL. Analysis of the mechanisms of expiratory asynchrony in pressure support ventilation: A mathematical approach. J Appl Physiol. 2000;88(6):2143-50.

10. Kacmarek RM. Proportional assist ventilation and neurally adjusted ventilatory assist. Respir Care. 2011;56(2):140-52.

11. Bosma KJ, Read BA, Bahrgard Nikoo MJ, Jones PM, Priestap FA, Lewis JF. A pilot randomized trial comparing weaning from mechanical ventilation on pressure support versus proportional assist ventilation. Crit Care Med. 2016;44(6):1098-108

12. Vaporidi K, Plataki M. Proportional assist ventilation with load-adjustable gain factors in critically ill patients: comparison with pressure support. 2008;2026-34.

13. Georgopoulos D, Xirouchaki N, Tzanakis N, Younes M. Driving pressure during assisted mechanical ventilation. Is it controlled by patient brain? Respir Physiol Neurobiol. 2016;228:69-75.

14. Kondili E, Prinianakis G, Alexopoulou C, Vakouti E, Klimathianaki M, Georgopoulos D. Respiratory load compensation during mechanical ventilation - Proportional assist ventilation with load-adjustable gain factors versus pressure support. Intensive Care Med. 2006;32(5):692-9.

15. Vasconcelos RS, Sales RP, Melo LH d P, Marinho LS, Bastos VPD, Nogueira A da NC, et al. Influences of duration of inspiratory effort, respiratory mechanics, and ventilator type on asynchrony with pressure support and proportional assist ventilation. Respir Care. 2017;62(5):550-7.

16. Carteaux G, Mancebo J, Mercat A, Dellamonica J, Richard J-CM, Aguirre HM, et al. An algorithm to adjust the percentage of assistance in $\mathrm{PAV}+$ based on an estimation of the patient's respiratory effort. 2010;292:A4085-A4085.

17. Akoumianaki E, Prinianakis G, Kondili E, Malliotakis P, Georgopoulos D. Physiologic comparison of neurally adjusted ventilator assist, proportional assist and pressure support ventilation in critically ill patients. Respir Physiol Neurobiol. 2014;203:82-9.

18. Kondili E, Alexopoulou C, Xirouchaki N, Vaporidi K, Georgopoulos D. Estimation of inspiratory muscle pressure in critically ill patients. Intensive Care Med. 2010;36(4):648-55
19. Carteaux G, Mancebo J, Mercat A, Dellamonica J, Richard JCM, Aguirre-Bermeo $\mathrm{H}$, et al. Bedside adjustment of proportional assist ventilation to target a predefined range of respiratory effort. Crit Care Med. 2013;41(9):2125-32.

20. Su PL, Kao PS, Lin WC, Su PF, Chen CW. Limited predictability of maximal muscular pressure using the difference between peak airway pressure and positive end-expiratory pressure during proportional assist ventilation (PAV). Crit Care. 2016;20(1):1-9.

21. Georgopoulos D, Xirouchaki N, Tzanakis N, Younes M. Data on respiratory variables in critically ill patients with acute respiratory failure placed on proportional assist ventilation with load adjustable gain factors (PAV+). Data Brief. 2016;8:484-93.

22. Vaporidi K, Psarologakis C, Proklou A, Pediaditis E, Akoumianaki E, Koutsiana $E$, et al. Driving pressure during proportional assist ventilation: an observational study. Ann Intensive Care. 2019;9(1):1-9.

23. Marantz S, Patrick W, Webster K, Roberts D, Oppenheimer L, Younes M. Response of ventilator-dependent patients to different levels of proportional assist. J Appl Physiol (1985). 1996;80(2):397-403.

24. Bosma K, Ferreyra G, Ambrogio C, Pasero D, Mirabella L, Braghiroli A et al. Patient-ventilator interaction and sleep in mechanically ventilated patients: Pressure support versus proportional assist ventilation. Crit Care Med. 2007;35(4):1048-54.

25. Grasso S, Puntillo F, Mascia L, Ancona G, Fiore T, Bruno F, et al. Compensation for increase in respiratory workload during mechanical ventilation: Pressure-support versus proportional-assist ventilation. Am J Respir Crit Care Med. 2000;161(3 I):819-26.

26. Ruiz-Ferrón F, Machado J, Morante A, Galindo S, Castillo A, Rucabado L. Trabajo y patrón respiratorio con diferentes niveles de ventilación asistida proporcional. Med Intensiva. 2009;33(6):269-75.

27. Ranieri VM, Giuliani R, Mascia L, Grasso S, Petruzzelli V, Puntillo N, et al. Patient-ventilator interaction during acute hypercapnia: pressure-support vs. proportional-assist ventilation. J Appl Physiol (1985). 1996;81(1):426-36

28. Parthasarathy $\mathrm{S}$, Tobin MJ. Sleep in the intensive care unit. Intensive Care Med. 2004;30(2):197-206.

29. Alexopoulou C, Kondili E, Vakouti E, Klimathianaki M, Prinianakis G, Georgopoulos D. Sleep during proportional-assist ventilation with load-adjustable gain factors in critically ill patients. Intensive Care Med. 2007:33(7):1139-47.

30. Georgopoulos D, Roussos C. Control of breathing in mechanically ventilated patients. Eur Respir J. 1996;9(10):2151-60.

31. Akoumianaki E, Dousse N, Lyazidi A, Lefebvre JC, Graf S, Cordioli RL, et al. Can proportional ventilation modes facilitate exercise in critically ill patients? A physiological cross-over study: Pressure support versus proportional ventilation during lower limb exercise in ventilated critically ill patients. Ann Intensive Care. 2017;7(1):64.

32. Romer LM, Polkey MI. Exercise-induced respiratory muscle fatigue: Implications for performance. J Appl Physiol. 2008;104(3):879-88.

33. Elganady AA, Beshey BN, Abdelaziz AAH, Alexopoulou C, Kondili E, Plataki $M$, et al. Is proportional-assist ventilation with load-adjustable gain factors a user-friendly mode? Intensive Care Med. 2010;35(9):2026-34.

34. Botha J, Green C, Carney I, Haji K, Gupta S, Tiruvoipati R. Proportional assist ventilation versus pressure support ventilation in weaning ventilation: a pilot randomised controlled trial. Crit Care Resusc. 2018;20(1): 33-40.

35. Teixeira SN, Osaku EF, Costa CR, et al. Comparison of Proportional Assist Ventilation Plus, T-Tube Ventilation, and Pressure Support Ventilation as Spontaneous Breathing Trials for Extubation: A Randomized Study. Respir Care. 2015;60(11):1527-1535.

36. Sasikumar S, Shanbhag V, Shenoy A. Comparison of pressure support ventilation and proportional assist ventilation plus for weaning from mechanical ventilation in critically ill patients. Ind J Resp Care 2013; 2: 292-8.

37. Xirouchaki N, Kondili E, Vaporidi K, Xirouchakis G, Klimathianaki M, Gavriilidis G, et al. Proportional assist ventilation with load-adjustable gain factors in critically ill patients: comparison with pressure support. Intensive Care Med. 2008;34(11):2026-34. 